\section{Modifying ADAMTS13 to modulate binding of pathogenic autoantibodies of patients with acquired thrombotic thrombocytopenic purpura}

\author{
Nuno A. G. Graça, ${ }^{1,2}$ Bogac Ercig, ${ }^{2,3,4}$ Leydi Carolina Velásquez Pereira, ${ }^{5}$ Kadri \\ Kangro, ${ }^{5}$ Paul Kaijen, ${ }^{2}$ Gerry A. F. Nicolaes, ${ }^{3,4}$ Agnès Veyradier, ${ }^{6,7}$ Paul Coppo, ${ }^{7-9}$ \\ Karen Vanhoorelbeke, ${ }^{5}$ Andres Männik ${ }^{1}$ and Jan Voorberg ${ }^{2}$ \\ ${ }^{1}$ Icosagen Cell Factory OÜ, Õssu, Kambja, Tartumaa, Estonia; ${ }^{2}$ Department of Molecular \\ and Cellular Hemostasis, Sanquin-Academic Medical Center Landsteiner Laboratory, \\ Amsterdam, the Netherlands; ${ }^{3}$ Pharmatarget, Maastricht, the Netherlands; ${ }^{4}$ Department \\ of Biochemistry, Cardiovascular Research Institute Maastricht (CARIM), Maastricht \\ University, Maastricht, the Netherlands; ${ }^{5}$ Laboratory for Thrombosis Research, IRF Life \\ Sciences, KU, Leuven Campus Kulak Kortrijk, Kortrijk, Belgium; 'Service d'Hématologie \\ Biologique and EA3518-Institut Universitaire d'Hématologie, Groupe Hospitalier Saint \\ Louis-Lariboisière, AP-HP, Université Paris Diderot, Paris, France; ${ }^{7}$ Centre de Référence \\ des Microangiopathies Thrombotiques, Hôpital Saint-Antoine, AP-HP, Paris, France; \\ ${ }^{8}$ Service d'Hématologie, Hôpital Saint-Antoine, AP-HP, Paris, France and 'Sorbonne \\ Université, UPMC Université Paris, Paris, France
}

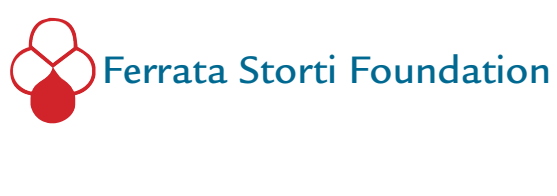

Haematologica 2020

Volume 105(11):2619-2630

\begin{abstract}
A ntibodies that develop in patients with immune thrombotic thrombocytopenic purpura commonly target the spacer epitope R568/F592/R660/Y661/Y665 (RFRYY) of ADAMTS13 (a disintegrin and metalloproteinase with a thrombospondin type 1 motif, member 13). In this study we present a detailed contribution of each residue in this epitope for autoantibody binding. Different panels of mutations were introduced to create a large collection of full-length ADAMTS13 variants comprising conservative $(\mathrm{Y} \leftarrow \mathrm{F})$, semi-conservative $(\mathrm{Y} / \mathrm{F} \rightarrow \mathrm{L})$, non-conservative $(\mathrm{Y} / \mathrm{F} \rightarrow \mathrm{N})$ or alanine $(\mathrm{Y} / \mathrm{F} / \mathrm{R} \rightarrow \mathrm{A})$ substitutions. Previously reported gain-of-function (KYKFF) and truncated 'MDTCS' variants were also included. Sera from 18 patients were screened against all variants. Conservative mutations of the aromatic residues did not reduce the binding of autoantibodies. Moderate resistance was achieved by replacing R568 and R660 by lysines or alanines. Semi-conservative mutations of aromatic residues showed a moderate effectiveness in autoantibody resistance. Non-conservative asparagine or alanine mutations of aromatic residues were the most effective. In the mixtures of autoantibodies from the majority (89\%) of patients screened, autoantibodies targeting the spacer RFRYY epitope were preponderant compared to other epitopes. Reductions in ADAMTS13 proteolytic activity were observed for all full-length mutant variants, in varying degrees. The greatest reductions in activity were observed in the most autoantibodyresistant variants (15-35\% residual activity in a FRETS-VWF73 assay). Among these, a triple-alanine mutant - RARAA - showed activity in a von Willebrand factor multimer assay. This study shows that non-conservative and alanine modifications of residues within the exosite- 3 spacer RFRYY epitope in full-length ADAMTS13 resist the binding of autoantibodies from patients with immune thrombotic thrombocytopenic purpura, while retaining residual proteolytic activity. Our study provides a framework for the design of autoantibody-resistant ADAMTS13 variants for further therapeutic development.
\end{abstract}

Partially presented as an oral communication at the $9^{\text {th }}$ Bari International Conference, Rome, Italy, September 17, 2017, and presented at the $10^{\text {th }}$ Bari International Conference, Genoa, Italy, September 8, 2019.

\section{Correspondence: \\ JAN VOORBERG \\ j.voorberg@sanquin.nl}

Received: May 8, 2019.

Accepted: November 21, 2019.

Pre-published: November 21, 2019.

doi:10.3324/haematol.2019.226068

(C)2020 Ferrata Storti Foundation

Material published in Haematologica is covered by copyright. All rights are reserved to the Ferrata Storti Foundation. Use of published material is allowed under the following terms and conditions:

https://creativecommons.org/licenses/by-nc/4.0/legalcode. Copies of published material are allowed for personal or internal use. Sharing published material for non-commercial purposes is subject to the following conditions: https://creativecommons. org//icenses/by-nc/4.0/legalcode, sect. 3. Reproducing and sharing published material for commercial purposes is not allowed without permission in writing from the publisher. 


\section{Introduction}

Thrombotic thrombocytopenic purpura (TTP) is a lifethreatening rare disorder triggered by a lack of activity of the von Willebrand factor (VWF)-cleaving protease ADAMTS13 (a disintegrin and metalloproteinase with a thrombospondin type 1 motif, member 13). A limited number of cases are congenital, however approximately $95 \%$ of cases are of an acquired, autoimmune nature immune TTP - in which autoantibodies targeting ADAMTS13 cause loss of enzyme activity resulting in accumulation of highly pro-thrombotic ultra-large VWF multimers. ${ }^{1}$ ADAMTS13 is a large, complex enzyme comprising 14 domains which, from the $\mathrm{N}$ - to C-terminal, are: metalloprotease (M), disintegrin (D), thrombospondintype 1-repeat 1 (TSP1), cysteine-rich (C), spacer (S), seven thrombospondin-type 1-repeats 2-8 (TSP2-8), and two CUB domains (CUB1 and CUB2) ${ }^{2}$ (Figure 1). The mechanisms for the loss of self-tolerance towards ADAMTS13 are not completely understood, but include specific HLA alleles, ethnicity and other genetic traits. ${ }^{3}$ Additionally, onset has also been associated with infections,${ }^{4,5}$ drugs, ${ }^{6}$ and cases of envenomation. ${ }^{7.8}$ Most autoantibodies of patients with immune TTP are encoded by the heavy chain variable region genes $\mathrm{V}_{\mathrm{H}} 1-69^{9 \cdot 12}$ and $\mathrm{V}_{\mathrm{H}} 1-3{ }^{12}$ However, the immune response is polyclonal, ${ }^{13}$ usually targeting the spacer domain, ${ }^{14}$ and may target other domains as well. ${ }^{15-17}$ Antibodies targeting the MDTCS domains physically block interactions between ADAMTS13 and VWF. ${ }^{18}$ So far, isolated autoantibodies against C-terminal TSP2-8 and CUB1-2 domains have not shown a clear direct inhibitory action, at least in static assays. ${ }^{10}$ Both types can increase the clearance of ADAMTS13, which is considered the major mechanism inducing loss of ADAMTS13 activity. ${ }^{17,19,20}$

Several epitope mapping studies revealed that in the exosite-3 of the spacer domain, an epitope comprising residues R568/F592/R660/Y661/Y665 (RFRYY) is commonly targeted in nearly $95 \%$ of patients with immune TTP (Online Supplementary Table S1). 10,15-17,21-24 Studies of spacer exosite-3 alanine scans have shown that alanine modifications lead to reductions in exosite- 3 spacer autoantibody binding, ${ }^{22,23}$ but also result in reductions of activity. ${ }^{23,25}$ Another study revealed that highly conserva-

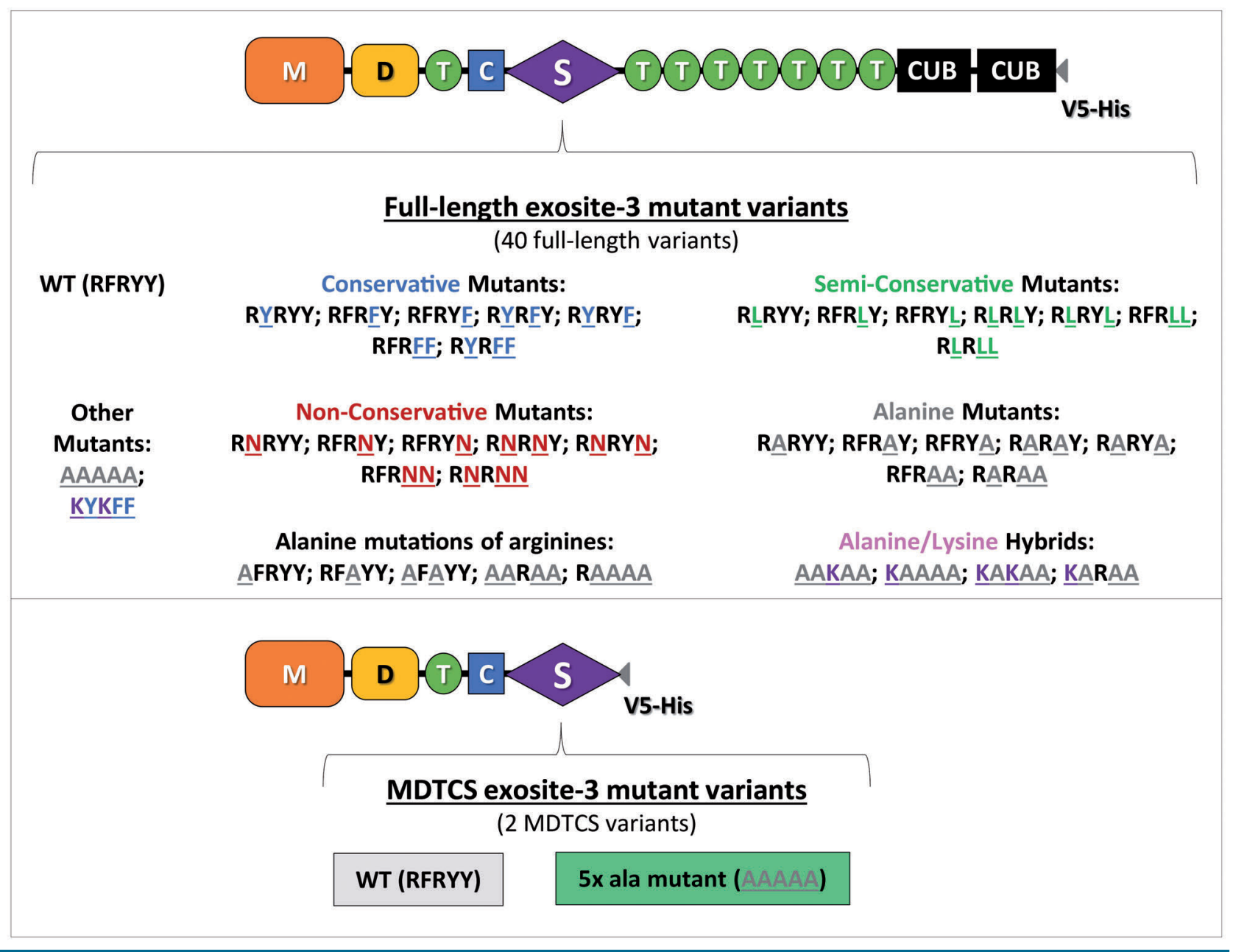

Figure 1. Overview of all ADAMTS13 spacer exosite-3 mutant variants created for this study. Full-length and MDTCS ADAMTS13 variants used in this study are shown. For ease and direct comparison with the original exosite-3 epitope (RFRYY), the mutations inserted are shown with the one-letter amino acid code in different colors and underlined. A total of 40 full-length variants were used, comprising conservative $(Y \leftrightarrow F)$, semi-conservative $(F / Y \rightarrow L)$, non-conservative $(F / Y \rightarrow N)$, classic alanine $(F / Y / R \rightarrow A)$ and alanine/lysine hybrid mutations $(F / Y \rightarrow A+R \rightarrow K)$. The residue substitutions were chosen based on the physicochemical characteristics of the amino acid side-chains (see Online Supplementary Methods for a more detailed description of the rationale for the different choices of amino acids). The classic gain-of-function (KYKFF) variant was also included in this study. In addition, two MDTCS variants were included (lower panel). 
tive substitutions inserted in a full-length context confer resistance to autoantibodies while generating a gain-offunction (GoF) ADAMTS13 with more activity than the wild-type form. ${ }^{26}$ These mutations were introduced cumulatively. At present, it is not clear how the degree of conservation of the amino-acid side-chains in the spacer exosite-3 RFRYY epitope affects both the autoantibody binding and the activity of ADAMTS13 variants.

In this study we present a refinement of the contribution of the residues in the exosite-3 RFRYY epitope to overall response of patients' autoantibodies. We included an extensive set of full-length spacer domain epitope variants with different degrees of residue conservation (Figure 1). The mutations inserted included single mutations and multiple progressively cumulative mutations, to scrutinize in more detail how they affect the binding of patients' autoantibodies. A total of 42 ADAMTS13 variants were screened for binding of autoantibodies from patients' samples. As expected, autoantibodies directed towards spacer domain exosite-3 constituted the most important subset of autoantibodies within the patients' response. We also demonstrated that within our panels of full-length ADAMTS13 variants, several displayed significantly reduced binding of autoantibodies while retaining residual proteolytic enzyme activity. Our study provides candidate molecules that could be used as a template for the design of novel therapeutic approaches that limit the binding of pathogenic autoantibodies to the spacer domain of ADAMTS13.

\section{Methods}

\section{Patients}

Patients' sera were obtained from the French Reference Center for Thrombotic Microangiopathies (CNR-MAT, Paris, France), from patients in the acute phase of onset of immune TTP, before treatment. All patients had ADAMTS13 activity $<10 \%$ (FRETS-VWF73) and detectable autoantibodies against ADAMTS13 in varying titers $(>15$ IU/mL, Technozyme ADAMTS13-INH ELISA kit; Technoclone, Vienna, Austria). All patients included were described in previous studies ${ }^{27,28}$ (Table 1). Informed consent was obtained from patients according to the Declaration of Helsinki. The study was approved by the Ethics Committee of Hôpital Pitié-Salpêtrière and Hôpital SaintAntoine (Assistance Publique-Hôpitaux de Paris, France).

\section{Assessment of reactivity of ADAMTS13 variants with patients' samples}

The design, construction, production, and computational modeling of human ADAMTS13 variants, as well as

Table 1. Patients' clinical data.

\begin{tabular}{|c|c|c|c|c|c|c|c|c|}
\hline Patient & Sex & Age & $\begin{array}{l}\text { ADAMIS13 } \\
\text { activity (FRETS- } \\
\text { WWF73, \%) }\end{array}$ & $\begin{array}{l}\text { IgG anti- } \\
\text { ADAMIS13 } \\
\text { (IU/mL) }\end{array}$ & $\begin{array}{l}\text { ADAMTS13 } \\
\text { antigen } \\
(\mu g / m L) *\end{array}$ & $\begin{array}{c}\text { Treatment regimen } \\
\text { in addition } \\
\text { to } \mathrm{PEX}^{8}\end{array}$ & $\begin{array}{l}\text { Detected } \\
\text { anti-spacer } \\
\text { idiotopes }\end{array}$ & $\begin{array}{l}\text { Cerebral } \\
\text { involvements }\end{array}$ \\
\hline TTP-007 & M & 55 & $<5$ & 100 & 0.05 & $\begin{array}{l}\text { + steroids + rituximab } \\
\text { + cyclophosphamide }\end{array}$ & Others & No \\
\hline TTP-008 & M & 74 & $<5$ & 120 & 0.05 & + steroids + rituximab & Others & Yes (confusion) \\
\hline TTP-012 & M & 55 & $<5$ & 260 & $<0.02$ & No treatment $\dagger$ & Others & No \\
\hline TTP-017 & $\mathrm{F}$ & 48 & $<5$ & 85 & $<0.02$ & + steroids + rituximab & Others & Yes (headaches) \\
\hline TTP-030 & $\mathrm{F}$ & 62 & $<5$ & 59 & 0.35 & PEX only & I-9 + II-1 + TTP73 & $\begin{array}{l}\text { Yes (aphasia) } \\
\text { idiotopes }\end{array}$ \\
\hline TTP-041 & $\mathrm{F}$ & 68 & $<5$ & 69 & 0.74 & + steroids + rituximab & II-1 + TTP73 idiotopes & Yes (seizure) \\
\hline TTP-042 & $\mathrm{F}$ & 45 & $<5$ & 69 & 0.16 & + steroids + rituximab & I-9 idiotope & $\begin{array}{l}\text { Yes (transient focal } \\
\text { defect) }\end{array}$ \\
\hline TTP-049 & $\mathrm{F}$ & 35 & $<5$ & 52 & 0.14 & + steroids & I-9 + TTP73 idiotopes & $\begin{array}{l}\text { Yes (headaches, } \\
\text { visual disorders) }\end{array}$ \\
\hline TTP-052 & $\mathrm{F}$ & 24 & $<5$ & 175 & 0.10 & + steroids + rituximab & $\begin{array}{c}\text { I-9 + II-1 + TTP73 } \\
\text { idiotopes }\end{array}$ & Yes (aphasia) \\
\hline TTP-057 & M & 52 & $<5$ & 87 & 0.11 & $\begin{array}{l}\text { + steroids + rituximab } \\
\text { + vincristine + splenectomy } \\
\text { (anti-VWF antibodies) }\end{array}$ & Others & No \\
\hline TTP-058 & $\mathrm{F}$ & 76 & $<5$ & $>100$ & 0.21 & + rituximab & Others & Yes (stroke) \\
\hline TTP-075 & M & 27 & $<5$ & 89 & 0.44 & + steroids & I-9 idiotope & Yes (headaches) \\
\hline TTP-076 & $\mathrm{F}$ & 45 & $<5$ & 65 & 0.09 & + steroids & I-9 + II-1 idiotopes & No \\
\hline TTP-079 & $\mathrm{F}$ & 37 & $<5$ & 180 & $<0.02$ & + steroids & I-9 + TTP73 idiotopes & No \\
\hline TTP-080 & M & 61 & $<10$ & 210 & $<0.02$ & PEX only & I-9 idiotope & No \\
\hline TTP-081 & M & 42 & $<5$ & 100 & 0.03 & + steroids + rituximab & $\begin{array}{l}\text { I-9 + II-1 + TTP73 } \\
\text { idiotopes }\end{array}$ & Yes (stroke) \\
\hline TTP-085 & $\mathrm{F}$ & 85 & $<5$ & $>100$ & $<0.02$ & + steroids & Others & $\begin{array}{l}\text { Yes (transient focal } \\
\text { defect) }\end{array}$ \\
\hline TTP-093 & $\mathrm{F}$ & 21 & $<5$ & 160 & 0.02 & + rituximab & Others & $\begin{array}{l}\text { Yes (headaches, } \\
\text { transient focal defect) }\end{array}$ \\
\hline
\end{tabular}

PEX: plasma exchange; M: male; F: female;VWF: von Willebrand factor; *Data from Roose et al.2018. ${ }^{27}{ }^{5}$ Data from Schelpe et al. $2019 .{ }^{28}$ 
expression and purification of anti-ADAMTS13 monoclonal antibodies I-9 and II-1 are given in the Online Supplementary Methods (see Online Supplementary Figures S1 and S2, and Online Supplementary Table S2). Figure 1 provides an overview of the variants that were created and included in this study. Several panels of conservative $(\mathrm{Y} \leftrightarrow \mathrm{F})$, semi-conservative $(\mathrm{Y} / \mathrm{F} \rightarrow \mathrm{L})$, and non-conservative $(\mathrm{Y} / \mathrm{F} \rightarrow \mathrm{N})$ mutations and alanine $(\mathrm{Y} / \mathrm{F} / \mathrm{R} \rightarrow \mathrm{A})$ substitutions, as well as a panel of alanine and lysine hybrids were employed in this study. For the non-conservative asparagine mutations no putative $\mathrm{N}$-glycosylation sites were introduced. The rationale for the choice of each type of mutation is presented in the Online Supplementary Methods. For ease, from here on throughout the text the variants will be referred to by the final mutated epitope with the residue replacement underlined (e.g., RFRYY is the wild-type and RFRLL is a double leucine mutant carrying Y661L/Y665L mutations). Murine antibodies 3H9 (anti-metalloprotease), and biotinylated 19H4 (anti-TSP8) and 17G2 (anti-CUB1) have been previously described..$^{29,30}$ Anti-V5 conjugated with horseradish peroxidase was obtained from Invitrogen ${ }^{\circledR}$ (Catalog \# R961-25).

To determine the binding of autoantibodies in patients' samples to the ADAMTS13 variants described, a sandwich enzyme-linked immunosorbent assay (ELISA) was developed in-house. Full details are provided in the Online Supplementary Methods. Briefly, antibody 3H9 was used to coat 96-well plates to capture ADAMTS13 from cell culture medium (coating done at $1 \mu \mathrm{g} / \mathrm{mL}$ ). Each patient's sample was tested for binding of autoantibodies against the variants described above. The samples were assessed against $200 \mathrm{ng} /$ well (1.05 nmol/well) of each ADAMTS13 full-length variant or against equal molar quantities of the MDTCS variants (78.75 ng/well), in duplicate. A pool of monoclonal antibodies against human-IgG1, IgG2, IgG3 and IgG4, each conjugated with horseradish peroxidase (Sanquin, the Netherlands) was used for detection. In each plate, a monoclonal antibody II-1 dilution curve against the full-length wild-type ADAMTS13 was fitted with a four-parameter fit model (GraphPad Prism 5.0). Serum reactivity (i.e., binding) against each ADAMTS13 variant was converted to II-1 equivalent units $(\mathrm{ng} / \mathrm{mL})$ through interpolation, and a ratio of the interpolated signals was expressed as a percentage of wild-type binding. A heat map with the average values obtained was used for data comparison (Figure 2). The median reactivity for each ADAMTS13 variant was calculated from its average reactivities against each patient's sample. An example of how the calculations were performed is given in Online Supplementary Figure S3.

\section{Assessment of the activity of ADAMTS13 variants}

All variants were assessed for activity through FRETSVWF73 assays, as previously described, ${ }^{31}$ using a Fluoroskan Ascent plate reader (Thermo Electron Corporation) with modifications. All FRETS assays were done using the supernatants of cell cultures, and a modified buffer was added to maintain the $\mathrm{pH}$ at $6.0^{32}$ (composition: $\mathrm{CaCl}_{2} 25 \mathrm{mM}$, Bis-Tris $20 \mathrm{mM}$, Tris- $\mathrm{HCl} 20 \mathrm{mM}$, HEPES $20 \mathrm{mM}$, Tween20 0.005\%). The recombinant fulllength wild-type ADAMTS13 was used as the calibrator for the assay (concentration range: 0.025-0.4 $\mu \mathrm{g} / \mathrm{mL}$ ). Supernatant from Chinese hamster ovary (CHO) cells not producing ADAMTS13 was included as a control. All variants were tested at the same molar concentration of 1.05
$\mathrm{nM}(0.2 \mu \mathrm{g} / \mathrm{mL}$ for full-length variants and $0.07875 \mu \mathrm{g} / \mathrm{mL}$ for MDTCS variants). An example of a calibration curve is shown in Online Supplementary Figure S4.

Selected variants were additionally tested for activity in a static VWF multimer assay as described in detail in the Online Supplementary Methods.

\section{Results}

The majority of autoantibodies in patients with immune thrombotic thrombocytopenic purpura target the spacer exosite-3 RFRYY epitope

All patients' samples were assessed for binding to each of the ADAMTS13 variants described with an in-house developed sandwich ELISA. Figure 2 shows the reactivity of all the different ADAMTS13 variants towards each patient's sample in a heat map format. To assess the potential presence of anti-TSP2-8 and CUB1-2 domain antibodies, we first compared the reactivity of the patients' samples with full-length wild-type ADAMTS13 and the MDTCS variants. Two patients (TTP-057 and 085) showed a loss of signal with wild-type MDTCS of more than $70 \%$. All other patients showed either similar signals to the full-length wild-type enzyme or, in a limited number of cases, losses up to $48 \%$. The MDTCS-AAAAA variant caused a major reduction in signal compared to wild-type MDTCS in all patients (with the exception of patient TTP-057). The same $5 x$ ala mutations in a fulllength context (AAAAA) caused large increases in signal for six patients compared to MDTCS $5 x$ ala (6/18; 33\%). These findings show that the autoantibodies targeting the spacer exosite-3 RFRYY epitope comprise the majority of the antibody mixture in most patients' autoantibody repertoire (16/18 cases, $89 \%)$. In rare cases, the repertoire was composed exclusively of autoantibodies targeting the TSP2-8 and/or CUB1-2 domains of ADAMTS13 (1/18 cases, $5 \%$ ). However, in $12 / 18$ cases $(67 \%)$ the repertoire was composed almost exclusively of autoantibodies targeting the RFRYY epitope in the spacer domain.

\section{Autoantibody resistance is obtained with non-conservative or alanine mutations}

Next we assessed the efficacy of each type of mutation at inducing autoantibody resistance. We designed variants that retained R568 and R660 intact, as well as variants in which these residues were mutated. Within the variants in which R568 and R660 were kept intact, variants containing conservative mutations of aromatic residues were still recognized by autoantibodies present in the patients' sera (Figure 2). Semi-conservative mutations in the spacer domain epitope showed reduced binding to patients' antibodies with signals in the range of $30 \%-75 \%$ when compared to the wild-type form in 15 patients, and below $30 \%$ in one patient. The non-conservative asparagine mutations were the most successful of the aromatic residue mutations at escaping binding by patients' autoantibodies, presenting signals within the range of $0 \%-75 \%$ for 16 patients. RFRNY and RFRYN mutants were the least successful of this panel at escaping the autoantibody response, followed by RFRNN. The RNRNN mutant had the lowest median reactivity of all aromatic residue triple mutants $(14 \%)$. Alanine mutations of aromatic residues

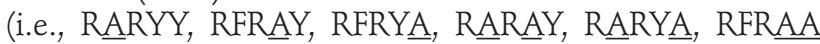
and RARAA) followed the same trend as the asparagine 


\begin{tabular}{|c|c|c|c|c|c|c|c|c|c|c|c|c|c|c|c|c|c|c|c|c|}
\hline \multirow{2}{*}{\multicolumn{2}{|c|}{$\begin{array}{l}\text { Mutant Variants } \\
\text { (exosite-3 residues) }\end{array}$}} & \multicolumn{18}{|c|}{ Patients tested } & \multirow{3}{*}{ 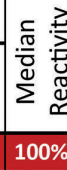 } \\
\hline & & \multirow{2}{*}{\begin{tabular}{|l} 
TTP \\
$\mathbf{0 0 7}$ \\
$100 \%$ \\
\end{tabular}} & \multirow{2}{*}{\begin{tabular}{|l|l|} 
TTP \\
$\mathbf{0 0 8}$ \\
$100 \%$
\end{tabular}} & \multirow{2}{*}{\begin{tabular}{|l|l} 
TTP \\
$\mathbf{0 1 2}$ \\
$100 \%$
\end{tabular}} & \multirow{2}{*}{\begin{tabular}{|l|l} 
TTP \\
$\mathbf{0 1 7}$ \\
$100 \%$
\end{tabular}} & \multirow{2}{*}{\begin{tabular}{|l|l|} 
TTP \\
$\mathbf{0 3 0}$ \\
$100 \%$
\end{tabular}} & \multirow{2}{*}{\begin{tabular}{|l|} 
TTP \\
$\mathbf{0 4 1}$ \\
$100 \%$
\end{tabular}} & \multirow{2}{*}{\begin{tabular}{|l|} 
TTP \\
$\mathbf{0 4 2}$ \\
$100 \%$
\end{tabular}} & \multirow{2}{*}{\begin{tabular}{|l|} 
TTP \\
$\mathbf{0 4 9}$ \\
$100 \%$
\end{tabular}} & \multirow{2}{*}{\begin{tabular}{|l|} 
TTP \\
$\mathbf{0 5 2}$ \\
$100 \%$ \\
\end{tabular}} & \multirow{2}{*}{\begin{tabular}{|l} 
TTP \\
$\mathbf{0 5 7}$ \\
$100 \%$
\end{tabular}} & \multirow{2}{*}{\begin{tabular}{|l|} 
TTP \\
$\mathbf{0 5 8}$ \\
$100 \%$ \\
\end{tabular}} & \multirow{2}{*}{\begin{tabular}{|l|l|} 
TTP \\
$\mathbf{0 7 5}$ \\
$100 \%$
\end{tabular}} & \begin{tabular}{|l} 
TTP \\
076
\end{tabular} & \begin{tabular}{|c|} 
TTP \\
079
\end{tabular} & $\begin{array}{l}\text { TTP } \\
080\end{array}$ & $\left|\begin{array}{l}\text { TTP } \\
081\end{array}\right|$ & $\begin{array}{l}\text { TTP } \\
085\end{array}$ & $\begin{array}{l}\text { TTP } \\
093\end{array}$ & \\
\hline & WT ful & & & & & & & & & & & & & $100 \%$ & $100 \%$ & $100 \%$ & $100 \%$ & $100 \%$ & $100 \%$ & \\
\hline & MDTCS W7 & $81 \%$ & $115 \%$ & $70 \%$ & $73 \%$ & $104 \%$ & $112 \%$ & $106 \%$ & $93 \%$ & $88 \%$ & $6 \%$ & $84 \%$ & $91 \%$ & $90 \%$ & $67 \%$ & $52 \%$ & $67 \%$ & $27 \%$ & $60 \%$ & $83 \%$ \\
\hline 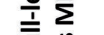 & MDTCS 5ala (A A A A A) & $4 \%$ & $6 \%$ & $20 \%$ & $8 \%$ & $0 \%$ & $0 \%$ & $20 \%$ & $7 \%$ & $1 \%$ & $0 \%$ & $0 \%$ & $1 \%$ & $0 \%$ & $10 \%$ & $11 \%$ & $2 \%$ & $1 \%$ & $4 \%$ & $3 \%$ \\
\hline & Full-length Mutant A A A A A & $10 \%$ & $7 \%$ & $77 \%$ & $20 \%$ & $0 \%$ & $0 \%$ & $19 \%$ & $6 \%$ & $4 \%$ & $90 \%$ & $2 \%$ & $0 \%$ & $0 \%$ & $40 \%$ & $49 \%$ & $27 \%$ & $101 \%$ & $8 \%$ & $9 \%$ \\
\hline & Mutant R $\underline{Y R} Y \mathbf{Y}$ & $89 \%$ & $101 \%$ & $102 \%$ & $83 \%$ & $101 \%$ & $106 \%$ & $105 \%$ & $92 \%$ & $104 \%$ & $106 \%$ & $92 \%$ & $107 \%$ & $84 \%$ & $81 \%$ & $88 \%$ & $78 \%$ & $105 \%$ & $103 \%$ & $101 \%$ \\
\hline & Mutant R F REY & $100 \%$ & $114 \%$ & $101 \%$ & $86 \%$ & $110 \%$ & $118 \%$ & $110 \%$ & $108 \%$ & $115 \%$ & $111 \%$ & $92 \%$ & $105 \%$ & $108 \%$ & $105 \%$ & $95 \%$ & $164 \%$ & $80 \%$ & $109 \%$ & $108 \%$ \\
\hline : & Mutant R F R Y & $98 \%$ & $117 \%$ & $118 \%$ & $98 \%$ & $128 \%$ & $108 \%$ & $107 \%$ & $100 \%$ & $112 \%$ & $100 \%$ & $95 \%$ & $118 \%$ & $104 \%$ & $115 \%$ & $94 \%$ & $142 \%$ & $91 \%$ & $114 \%$ & $108 \%$ \\
\hline$?$ & Mutant R $\underline{Y} \mathbf{R} \underline{\mathrm{F}} \mathbf{Y}$ & $87 \%$ & $104 \%$ & $107 \%$ & $86 \%$ & $110 \%$ & $109 \%$ & $109 \%$ & $87 \%$ & $108 \%$ & $102 \%$ & $77 \%$ & $111 \%$ & $79 \%$ & $75 \%$ & $87 \%$ & $135 \%$ & $99 \%$ & $105 \%$ & $103 \%$ \\
\hline & Mutant R $\underline{Y} \mathbf{R} Y \underline{F}$ & $89 \%$ & $96 \%$ & $121 \%$ & $82 \%$ & $101 \%$ & $114 \%$ & $111 \%$ & $96 \%$ & $116 \%$ & $112 \%$ & $93 \%$ & $103 \%$ & $62 \%$ & $70 \%$ & $92 \%$ & $100 \%$ & $121 \%$ & $107 \%$ & $100 \%$ \\
\hline & $\underline{F F}$ & $87 \%$ & $103 \%$ & $100 \%$ & $92 \%$ & $121 \%$ & $112 \%$ & $103 \%$ & $76 \%$ & $109 \%$ & $93 \%$ & $84 \%$ & $100 \%$ & $110 \%$ & $101 \%$ & $86 \%$ & $187 \%$ & $83 \%$ & $110 \%$ & $101 \%$ \\
\hline & $\mathrm{F}$ & $83 \%$ & $102 \%$ & $114 \%$ & $93 \%$ & $67 \%$ & $105 \%$ & $99 \%$ & $86 \%$ & $92 \%$ & $106 \%$ & $87 \%$ & $102 \%$ & $59 \%$ & $64 \%$ & $102 \%$ & $85 \%$ & $93 \%$ & $89 \%$ & $92 \%$ \\
\hline & Mutant $R\lfloor R Y Y$ & $89 \%$ & $114 \%$ & $110 \%$ & $80 \%$ & $105 \%$ & $104 \%$ & $98 \%$ & $95 \%$ & $101 \%$ & $124 \%$ & $90 \%$ & $104 \%$ & $62 \%$ & $54 \%$ & $75 \%$ & $62 \%$ & $79 \%$ & $86 \%$ & $93 \%$ \\
\hline & Mutant R F R $\underline{\underline{ } \mathbf{Y}}$ & $90 \%$ & $108 \%$ & $110 \%$ & $91 \%$ & $102 \%$ & $105 \%$ & $100 \%$ & $102 \%$ & $98 \%$ & $119 \%$ & $95 \%$ & $111 \%$ & $65 \%$ & $74 \%$ & $90 \%$ & $82 \%$ & $81 \%$ & $94 \%$ & $97 \%$ \\
\hline & $\underline{\underline{L}}$ & $96 \%$ & $119 \%$ & $119 \%$ & $95 \%$ & $120 \%$ & $109 \%$ & $101 \%$ & $103 \%$ & $113 \%$ & $120 \%$ & $101 \%$ & $117 \%$ & $100 \%$ & $107 \%$ & $96 \%$ & $132 \%$ & $81 \%$ & $101 \%$ & $105 \%$ \\
\hline & Muta & $71 \%$ & $92 \%$ & $101 \%$ & $58 \%$ & $71 \%$ & $70 \%$ & $97 \%$ & $83 \%$ & $85 \%$ & $119 \%$ & $67 \%$ & $85 \%$ & $19 \%$ & $9 \%$ & $62 \%$ & $53 \%$ & $74 \%$ & $76 \%$ & $72 \%$ \\
\hline & Mutant $R \underline{\underline{L}} \mathbf{R} \mathrm{Y} \underline{\underline{\mathrm{L}}}$ & $81 \%$ & $117 \%$ & $104 \%$ & $67 \%$ & $98 \%$ & $108 \%$ & $105 \%$ & $94 \%$ & $101 \%$ & $125 \%$ & $75 \%$ & $101 \%$ & $53 \%$ & $63 \%$ & $80 \%$ & $73 \%$ & $85 \%$ & $93 \%$ & $93 \%$ \\
\hline & Mutant R F R $\underline{\underline{L} \underline{L}}$ & $78 \%$ & $99 \%$ & $102 \%$ & $82 \%$ & $94 \%$ & $106 \%$ & $100 \%$ & $96 \%$ & $106 \%$ & $114 \%$ & $87 \%$ & $96 \%$ & $53 \%$ & $57 \%$ & $90 \%$ & $95 \%$ & $102 \%$ & $97 \%$ & $96 \%$ \\
\hline & $\mathbf{N}$ & $1 \%$ & $67 \%$ & $78 \%$ & $51 \%$ & $72 \%$ & 63\% & $98 \%$ & $64 \%$ & $73 \%$ & $112 \%$ & $47 \%$ & $72 \%$ & $14 \%$ & $19 \%$ & $55 \%$ & $53 \%$ & $64 \%$ & $73 \%$ & $64 \%$ \\
\hline & $\mathrm{N}$ & $60 \%$ & $80 \%$ & $110 \%$ & $7 \%$ & $61 \%$ & $63 \%$ & $91 \%$ & $71 \%$ & $73 \%$ & $109 \%$ & $61 \%$ & $67 \%$ & $14 \%$ & $45 \%$ & $77 \%$ & $52 \%$ & $81 \%$ & $57 \%$ & $65 \%$ \\
\hline & Mutant R F R $\underline{\mathrm{N}} \mathrm{Y}$ & $73 \%$ & $82 \%$ & $98 \%$ & $56 \%$ & $73 \%$ & $81 \%$ & $94 \%$ & $78 \%$ & $79 \%$ & $87 \%$ & $74 \%$ & $79 \%$ & $33 \%$ & $51 \%$ & $64 \%$ & $53 \%$ & $89 \%$ & $68 \%$ & $76 \%$ \\
\hline & Muta & $92 \%$ & $106 \%$ & $128 \%$ & $83 \%$ & $96 \%$ & $119 \%$ & $99 \%$ & $103 \%$ & $118 \%$ & $87 \%$ & $98 \%$ & $108 \%$ & $84 \%$ & $98 \%$ & $89 \%$ & $97 \%$ & $102 \%$ & $91 \%$ & $98 \%$ \\
\hline & Mut & $28 \%$ & $33^{\circ}$ & $105 \%$ & $19 \%$ & $28 \%$ & $11 \%$ & $73 \%$ & $6 \%$ & $30 \%$ & $85 \%$ & $25 \%$ & $27 \%$ & $0 \%$ & $55 \%$ & $56 \%$ & $43 \%$ & $86 \%$ & $27 \%$ & $32 \%$ \\
\hline & Muta & $32 \%$ & $52 \%$ & $113 \%$ & $26 \%$ & $49 \%$ & $25 \%$ & $74 \%$ & $44 \%$ & $47 \%$ & $60 \%$ & $39 \%$ & $36 \%$ & $2 \%$ & $66 \%$ & $64 \%$ & $46 \%$ & $98 \%$ & $40 \%$ & $47 \%$ \\
\hline & Mutant R F R N N & $57 \%$ & $74 \%$ & $97 \%$ & $38 \%$ & $54 \%$ & $55 \%$ & $88 \%$ & $66 \%$ & $69 \%$ & $67 \%$ & $65 \%$ & $68 \%$ & $15 \%$ & $60 \%$ & $55 \%$ & & $95 \%$ & $50 \%$ & $62 \%$ \\
\hline & Mutant R $\underline{\mathrm{N}} \mathrm{R} \underline{\mathrm{N}}$ & $11 \%$ & $10 \%$ & $88 \%$ & $18 \%$ & $8 \%$ & $0 \%$ & $47 \%$ & $16 \%$ & $9 \%$ & $57 \%$ & $6 \%$ & $10 \%$ & $1 \%$ & $51 \%$ & $51 \%$ & $41 \%$ & $98 \%$ & $10 \%$ & $14 \%$ \\
\hline & $\operatorname{tant} R A R Y Y$ & $56 \%$ & $65 \%$ & $60 \%$ & $43 \%$ & $51 \%$ & $56 \%$ & $84 \%$ & $58 \%$ & $58 \%$ & $95 \%$ & $56 \%$ & $55 \%$ & $4 \%$ & $26 \%$ & $38 \%$ & $45 \%$ & $54 \%$ & $44 \%$ & $55 \%$ \\
\hline & Mutar & $74 \%$ & $98 \%$ & $119 \%$ & $67 \%$ & $72 \%$ & $87 \%$ & $94 \%$ & $87 \%$ & $88 \%$ & $99 \%$ & $88 \%$ & $90 \%$ & $38 \%$ & $56 \%$ & $61 \%$ & $60 \%$ & $85 \%$ & $79 \%$ & $86 \%$ \\
\hline & Mutant R F R Y $\underline{A}$ & $87 \%$ & $109 \%$ & $115 \%$ & $76 \%$ & $87 \%$ & $118 \%$ & $99 \%$ & $99 \%$ & $105 \%$ & $86 \%$ & $105 \%$ & $109 \%$ & $72 \%$ & $89 \%$ & $71 \%$ & $56 \%$ & $101 \%$ & $95 \%$ & $97 \%$ \\
\hline & Mutant R A R A Y Y & & $52 \%$ & $86 \%$ & $29 \%$ & $33 \%$ & $27 \%$ & $78 \%$ & $47 \%$ & $47 \%$ & $99 \%$ & $2 \%$ & $48 \%$ & $0 \%$ & $51 \%$ & $41 \%$ & $33 \%$ & $85 \%$ & $44 \%$ & $45 \%$ \\
\hline & Mutant RARYA & $49 \%$ & $65 \%$ & $77 \%$ & $39 \%$ & $46 \%$ & $66 \%$ & $82 \%$ & $67 \%$ & $69 \%$ & $110 \%$ & $57 \%$ & $54 \%$ & $6 \%$ & $40 \%$ & $48 \%$ & $31 \%$ & $82 \%$ & $65 \%$ & $61 \%$ \\
\hline & Mutant R F R A A & $54 \%$ & $80 \%$ & $87 \%$ & $42 \%$ & $51 \%$ & $65 \%$ & $92 \%$ & $73 \%$ & $84 \%$ & $73 \%$ & $75 \%$ & $73 \%$ & $20 \%$ & $65 \%$ & $48 \%$ & $35^{\circ}$ & $99 \%$ & $65 \%$ & $69 \%$ \\
\hline$i$ & Mutant A F R Y Y & $70 \%$ & $94 \%$ & $85 \%$ & $77 \%$ & $66 \%$ & $103 \%$ & $90 \%$ & $100 \%$ & $103 \%$ & $86 \%$ & $92 \%$ & $98 \%$ & $91 \%$ & $96 \%$ & $82 \%$ & $78 \%$ & $112 \%$ & $87 \%$ & $91 \%$ \\
\hline & Mutant R F A Y Y & $57 \%$ & $90 \%$ & $83 \%$ & $68 \%$ & $76 \%$ & $90 \%$ & $86 \%$ & $88 \%$ & $101 \%$ & $93 \%$ & $85 \%$ & $82 \%$ & $83 \%$ & $99 \%$ & $83 \%$ & $94 \%$ & $113 \%$ & $86 \%$ & $86 \%$ \\
\hline & Mutant $A F A Y Y$ & $32 \%$ & $72 \%$ & $65 \%$ & $49 \%$ & $54 \%$ & $67 \%$ & $62 \%$ & $63 \%$ & $78 \%$ & $77 \%$ & $69 \%$ & $60 \%$ & $63 \%$ & $90 \%$ & $62 \%$ & 61. & $105 \%$ & $70 \%$ & $64 \%$ \\
\hline & Mutant R A R A A & $26 \%$ & $35 \%$ & $72 \%$ & $24 \%$ & $10 \%$ & $5 \%$ & $57 \%$ & $28 \%$ & $25 \%$ & $94 \%$ & $17 \%$ & $26 \%$ & $3 \%$ & $44 \%$ & $47 \%$ & $34 \%$ & $83 \%$ & $24 \%$ & $27 \%$ \\
\hline g & Mutant A A R A A & $7 \%$ & $17 \%$ & $91 \%$ & $22 \%$ & $10 \%$ & $1 \%$ & $28 \%$ & $21 \%$ & $12 \%$ & $72 \%$ & $9 \%$ & $10 \%$ & $0 \%$ & $66 \%$ & $47 \%$ & 35\% & $136 \%$ & $26 \%$ & $21 \%$ \\
\hline (c) & Mutant RAA A A & $10 \%$ & $15 \%$ & $85 \%$ & $29 \%$ & $27 \%$ & $8 \%$ & $31 \%$ & $20 \%$ & $14 \%$ & $96 \%$ & $14 \%$ & $9 \%$ & $2 \%$ & $61 \%$ & $40 \%$ & $22 \%$ & $155 \%$ & $31 \%$ & $24 \%$ \\
\hline & Full-length Mutant $\mathrm{A} A \mathrm{~A} A \mathrm{~A} A$ & $10 \%$ & $7 \%$ & $77 \%$ & $20 \%$ & $0 \%$ & $0 \%$ & $19 \%$ & $6 \%$ & $4 \%$ & $90 \%$ & $2 \%$ & $0 \%$ & $0 \%$ & $40 \%$ & $49 \%$ & $27 \%$ & $101 \%$ & $8 \%$ & $9 \%$ \\
\hline & Mutant A A K A A & $7 \%$ & $14 \%$ & $75 \%$ & $21 \%$ & $7 \%$ & $0 \%$ & $26 \%$ & $19 \%$ & $14 \%$ & $63 \%$ & $11 \%$ & $9 \%$ & $1 \%$ & $60 \%$ & $62 \%$ & $31 \%$ & $119 \%$ & $19 \%$ & $19 \%$ \\
\hline & Mutant K A A A A & $9 \%$ & $13 \%$ & $79 \%$ & $23 \%$ & $12 \%$ & $0 \%$ & $28 \%$ & $27 \%$ & $14 \%$ & $98 \%$ & $12 \%$ & $9 \%$ & $1 \%$ & $65 \%$ & $54 \%$ & 34: & $127 \%$ & $25 \%$ & $24 \%$ \\
\hline & Mutant KA KAA & $10 \%$ & $23 \%$ & $81 \%$ & $20 \%$ & $10 \%$ & $1 \%$ & $36 \%$ & $27 \%$ & $20 \%$ & $73 \%$ & $16 \%$ & $18 \%$ & $0 \%$ & $72 \%$ & $56 \%$ & 35: & $131 \%$ & $30 \%$ & $25 \%$ \\
\hline & Mutant KA R A A & $14 \%$ & $34 \%$ & $84 \%$ & $22 \%$ & $0 \%$ & $6 \%$ & $43 \%$ & $33 \%$ & $22 \%$ & $82 \%$ & $17 \%$ & $25 \%$ & $1 \%$ & $71 \%$ & $57 \%$ & 38\% & $129 \%$ & $41 \%$ & $34 \%$ \\
\hline GoF & Mutant KY Y K F F & $58 \%$ & $84 \%$ & $68 \%$ & $60 \%$ & $82 \%$ & $84 \%$ & $89 \%$ & $73 \%$ & $94 \%$ & $76 \%$ & $88 \%$ & $85 \%$ & $49 \%$ & $52 \%$ & $62 \%$ & $56 \%$ & $64 \%$ & $73 \%$ & $73 \%$ \\
\hline & rotein Supernatant & $0 \%$ & $0 \%$ & $0 \%$ & $0 \%$ & $7 \%$ & $0 \%$ & $0 \%$ & $1 \%$ & $0 \%$ & $0 \%$ & $0 \%$ & $0 \%$ & $0 \%$ & $0 \%$ & $0 \%$ & $0 \%$ & $0 \%$ & $0 \%$ & $0 \%$ \\
\hline Dilu & $\begin{array}{l}\text { Factor for each patient } \\
\text { (fold dilution) }\end{array}$ & ๖ & ঠัષ & ర્ఠ & ঠ্ব & Ð্ল & ১্ব & ১্ব & ఫ্ল & ১্ণ & ত্ব & ర্ট & ১্ব & ১্ণ & 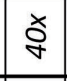 & ๖્ఫ & ১্ & ๖̀ & ఠ & \\
\hline Aut & antibodies titer (I.U./mL) & 100 & 120 & 260 & 85 & 59 & 69 & 69 & 52 & 175 & 87 & $>100$ & 89 & 65 & 180 & 210 & 100 & $>100$ & 160 & \\
\hline
\end{tabular}

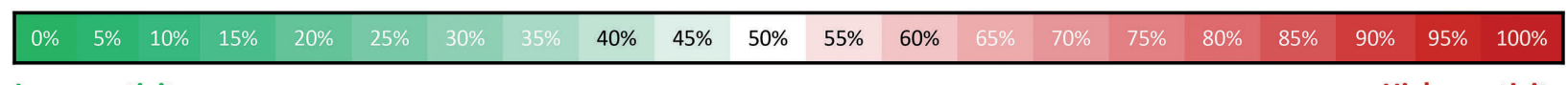

\section{Ratio mutant variant reactivity / full-length WT reactivity (\%)}

Figure 2. Heat map with reactivity of all the patients' samples tested against all ADAMTS13 spacer exosite-3 RFRYY mutant variants created for this study. Mutants (left) are organized by mutation type with residues mutated in color and underlined (blue: conservative mutations; green: semi-conservative mutations; red: non-conservative mutations; gray: alanine mutations; purple: arginine-to-lysine mutations), and by number of mutations introduced. The classic gain-of-function (GoF) variant is displayed separately for comparison. Patients' dilution factors and antibody titers are indicated in the rows below. The median reactivity of each mutant is displayed in the same heat-map fashion (right column). A color scale for reactivity is displayed below with green backgrounds indicating low reactivity, white backgrounds inter mediate reactivity and red backgrounds high reactivity $(n=2)$. 
mutations, and were also effective at escaping binding of patients' antibodies, albeit to a lesser degree. The RARAA mutant had the second lowest median reactivity with patients' antibodies among this panel of alanine substitutions $(27 \%)$. For both asparagine and alanine mutations, the more cumulative mutations were introduced, the higher was the effectiveness at escaping autoantibody binding.

Within the panels in which the R568 and R660 were also mutated, mutation of the arginines to alanines alone had a moderate effect on autoantibody resistance (median signal at $64 \%$ for mutant AFAYY) (Figure 2). The classic GoF molecule (KYKFF) also presented a moderate decrease in binding to patients' antibodies when compared to the wild-type form and to other mutations introduced. The median reactivity of the GoF variant was $73 \%$, a mild improvement compared to a similar conservative mutant (RYRFF: median reactivity: 92\%). As can be deduced from the RARAA mutant, addition of a further arginine mutation with alanine (AARAA and RAAAA mutants) promoted a further, but more limited, reduction of median reactivity ( $21 \%$ and $24 \%$, respectively). Further mutating the remaining arginines to lysines (i.e., AAKAA, KAAAA, KAKAA mutants) produced little to no variation in median reactivity (19\%-25\%), with the KARAA mutant showing a small increase when compared to KAKAA. The 5x ala full-length mutant (AAAAA) had the greatest decrease in median reactivity among all full-length variants (median signal: 9\%). Our findings show that cumulative replacement of aromatic residues in the spacer RFRYY epitope of ADAMTS13 with asparagine or alanine provides superior resistance towards immune TTP patients' autoantibodies directed towards the spacer domain.

\section{Autoantibody-resistant ADAMTS13 variants retain proteolytic activity}

We subsequently assessed the impact of these mutations on the activity of ADAMTS13. All variants were tested in a FRETS-VWF73 assay at the same molar concentration and directly compared with the full-length wildtype ADAMTS13 (Online Supplementary Figure S4). The relative activities are shown in Figure 3. All full-length variants showed reduced activity in FRETS-VWF73 when compared to the full-length wild-type ADAMTS13. The degree of reduction varied depending on the mutations inserted. Conservative mutations retained the most activity on average. The RYRYY mutant had 39\% activity while RFRFF had $76 \%$. The triple conservative RYRFF mutant retained $56 \%$ of activity. Semi-conservative leucine mutants also retain overall high activity, with single and double mutations retaining between 35\% and $76 \%$ activity. The RLRLL mutant had a larger loss of activ-

A

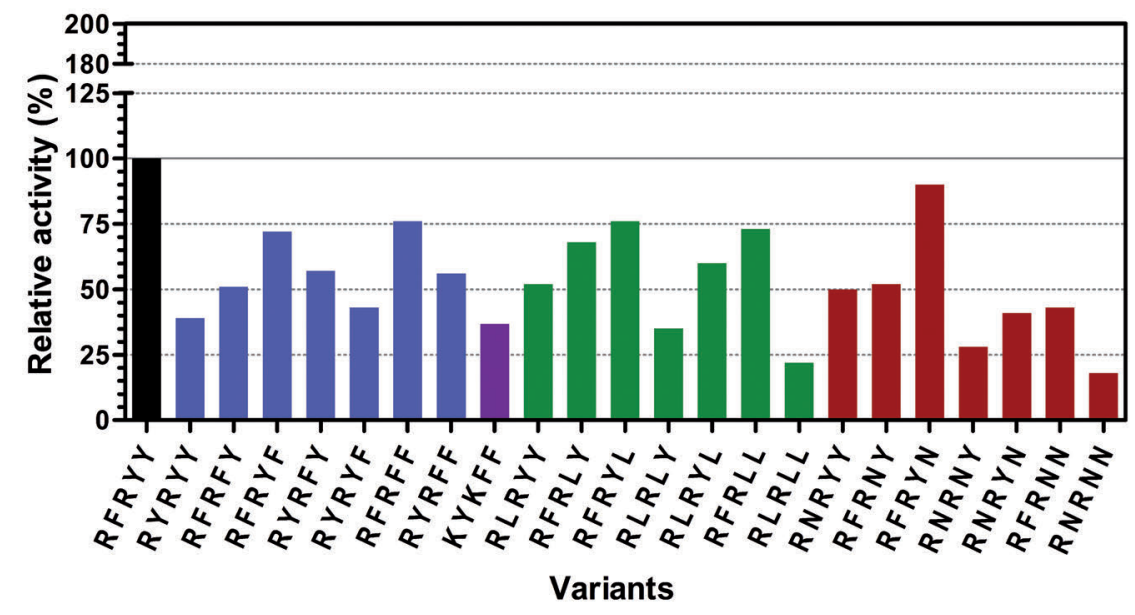

B

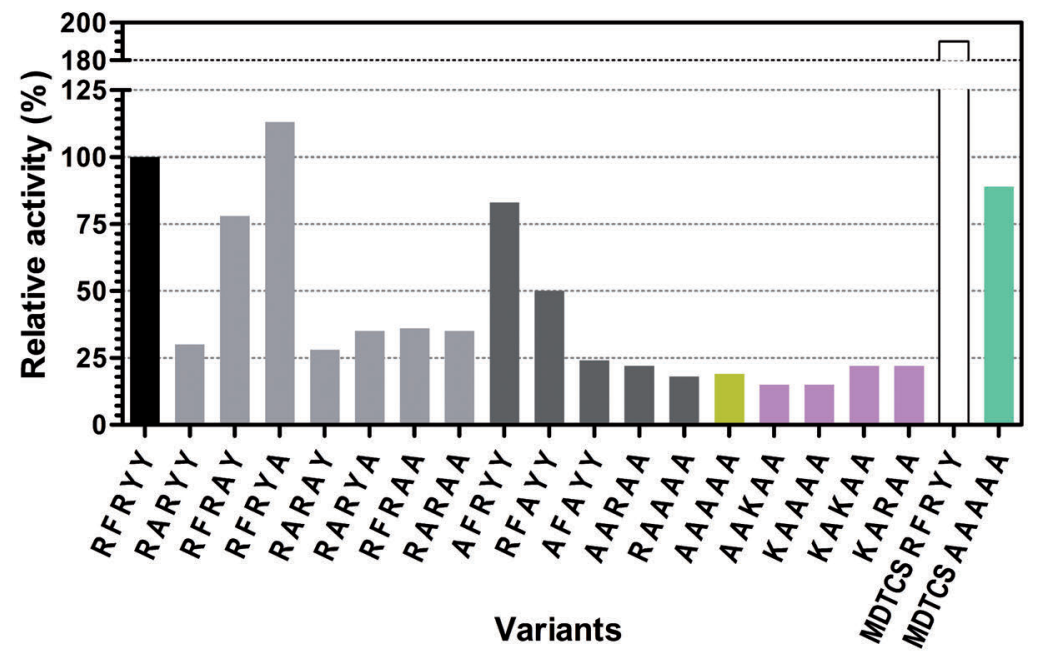

Figure 3. FRETS-VWF73 activity data of mutant ADAMTS13 variants. All mutant variants in this study were assessed for activity using a FRETS-VWF73 assay. Three independent assays were conducted, each one with its own calibration curve constructed using the recombinant fulllength wild-type ADAMTS13 (an example of a calibration curve is shown in Online Supplementary Figure S4). In each assay, a limited number of variants could be introduced. All values are normalized to the full-length wildtype molecule (100\% activity). (A) The relative activities of the variants are shown. The fulllength wild-type ADAMTS13 is represented in black, the conservative variants in blue, the gain-of-function in purple, semiconservative variants in green and non-conservative variants in red. (B) The relative activities of variants are shown. The fulllength wild-type ADAMTS13 is represented in black, the aromatic-alanine variants in light gray, the arginine-alanine variants in dark gray, the full-length AAAAA in golden, the alanine/lysine hybrids in pink, the wild-type MDTCS in white and the MDTCS-AAAAA mutant in green. 
ity, retaining only $22 \%$. Single asparagine and alanine mutations of aromatic residues induced limited losses of activity. Cumulative double and triple asparagine or alanine mutations had more detrimental effects, with alanine mutations having less negative effects (RNRNN: 18\%; RARAA: $35 \%$ ). Mutation of the arginines by alanines had different impacts: R660A (RFAYY) had a higher reduction of activity (50\% activity retained) compared to R568A (AFRYY) (83\% activity retained). Mutation of both arginines (AFAYY) caused a large reduction of activity (24\% that of the wild-type molecule). The classic GoF molecule (KYKFF) followed all trends observed for conservative mutations combined with arginine mutations and, surprisingly, also displayed reduced activity compared to the wild-type form ( $37 \%$ activity retained). The fulllength mutants AAAAA, AARAA, RAAAA, AAKAA, KAAAA, KAKAA, and KARAA all had similar activities (15-22\%). The MDTCS variants displayed higher activities than each of their full-length counterparts (wild-type MDTCS with 190\% and MDTCS-AAAAA with $89 \%$ vs. $100 \%$ and $19 \%$, respectively). In general, an inverse correlation was observed: a higher resistance to autoantibody binding attained through spacer exosite- 3 mutations resulted, in general, in a lower residual FRETS-VWF73 activity (Figure 4).

The activities of five variants were tested in the presence of patients' autoantibodies in a FRETS-VWF73 assay format (Figure 5). Samples from patients TTP-007, TTP-
008 (exclusively anti-spacer autoantibodies) and patient TTP-085 (anti-spacer and anti-C-terminal autoantibodies) were used to provide a proof-of-concept. While the wildtype and the classic GoF were clearly inhibited, all other selected variants retained the same levels of activity.

Subsequently, several mutants with different levels of FRETS-VWF73 activities were tested in a static VWF multimer assay (Figure 6). The wild-type molecule showed activity after $30 \mathrm{~min}$, with high molecular weight VWF multimers being cleaved and satellite bands accumulating in the lower molecular weight regions. After $24 \mathrm{~h}$, these differences were further pronounced. All of the mutants tested in this assay showed reduced activity compared to the wild-type molecule. The classic GoF (KYKFF) demonstrated the highest activity among all the mutants tested. Among the autoantibody-resistant mutants, RARAA had the highest activity, with a reduction of high molecular weight VWF multimers and accumulation of satellite bands. While the truncated wild-type MDTCS variant had equal activity to full-length wild-type ADAMTS13, the MDTCS-AAAAA variant had very low activity, comparable to that of its full-length counterpart,

\section{Discussion}

In the current study we assessed the relevance of the spacer RFRYY epitope residues for autoantibody binding,

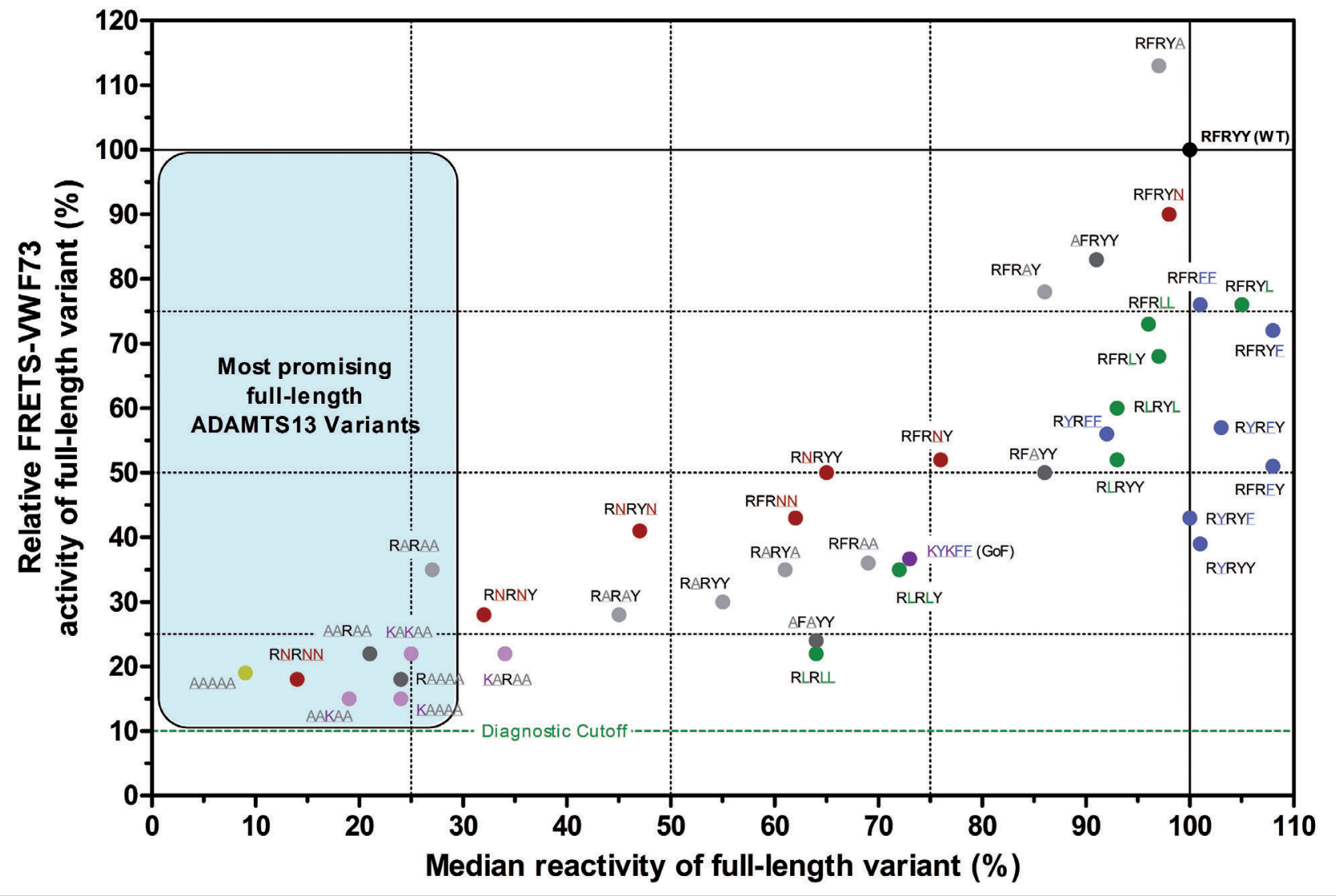

Figure 4. Inverse correlation observed between autoantibody reactivity and activity of ADAMTS13 variants. Only full-length ADAMTS13 variants are shown. The cyanblue shading shows the area in which ADAMTS13 variants with reduced autoantibody binding are displayed. Blue dots represent conservative mutants; green dots represent semi-conservative mutants; red dots represent non-conservative mutants; gray dots represent alanine mutants (light-gray for only aromatic residues; darkgray for arginine mutants); pink represents lysine/alanine hybrids. 
and the types of mutations that confer highest resistance to binding of patients' anti-ADAMTS13 autoantibodies. In our study, the spacer RFRYY epitope made a major contribution to total autoantibody reactivity in $89 \%$ of the patients with immune TTP screened. Other studies reached similar conclusions. ${ }^{17,18}$ In our study, 12/18 patients $(67 \%)$, had autoantibody mixtures comprising almost exclusively anti-spacer RFRYY autoantibodies. We also had $6 / 18$ patients (33\%) who had C-terminal antiTSP2-8 and/or anti-CUB1-2 domain autoantibodies, a prevalence slightly below the range reported in the literature (Online Supplementary Table S1). One patient (TTP042) also had autoantibodies targeting other unknown areas in the $\mathrm{N}$-terminal region (Figure 2).

We show that different types of mutations in ADAMTS13 spacer exosite-3 have different impacts on immune TTP patients' autoantibody binding. Our data indicate that the two arginines are less important for autoantibody binding than the three aromatic residues present in this epitope (AFAYY had a median reactivity of $64 \%$, while RARAA had only $27 \%$ ). Conservative mutations of aromatic residues did not reduce reactivity towards patients' autoantibodies (RYRFF median reactivity: $92 \%$ ). Replacing the arginines in this mutant by lysines (KYKFF, classic GoF mutant) led to a decrease in median reactivity (in agreement with the original study ${ }^{26}$ ), albeit quantitatively limited (73\% binding). Thus, introduction of highly conservative mutations of aromatic residues is not sufficient to reduce the binding of autoantibodies targeting the RFRYY epitope. Semi-conservative mutations of aromatic residues with leucine had an intermediate, yet limited efficacy at autoantibody escape. Non-conservative asparagine or classic alanine mutations of the aromatic residues had the greatest success. Individual mutations had a low impact, and, by themselves, Y661 and Y665 appeared to be the least important residues for overall autoantibody binding. A more pivotal contribution was observed for F592, as shown when individually or cumu-

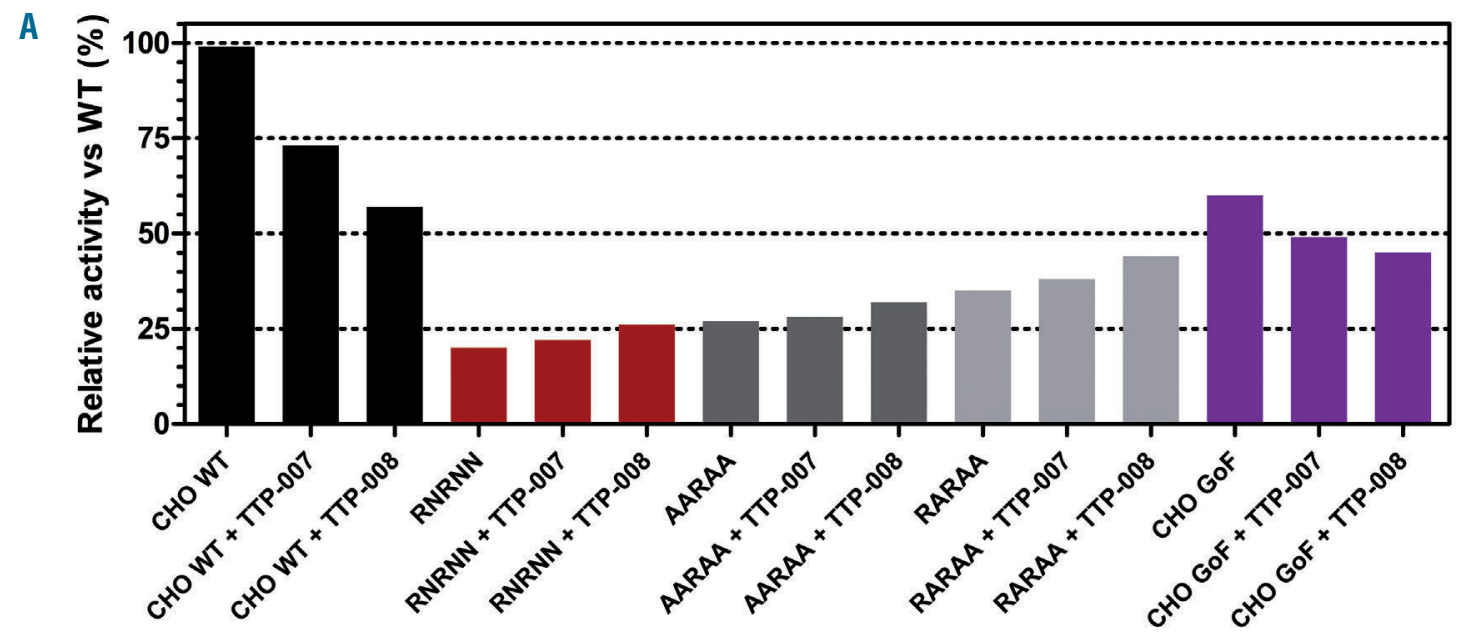

B

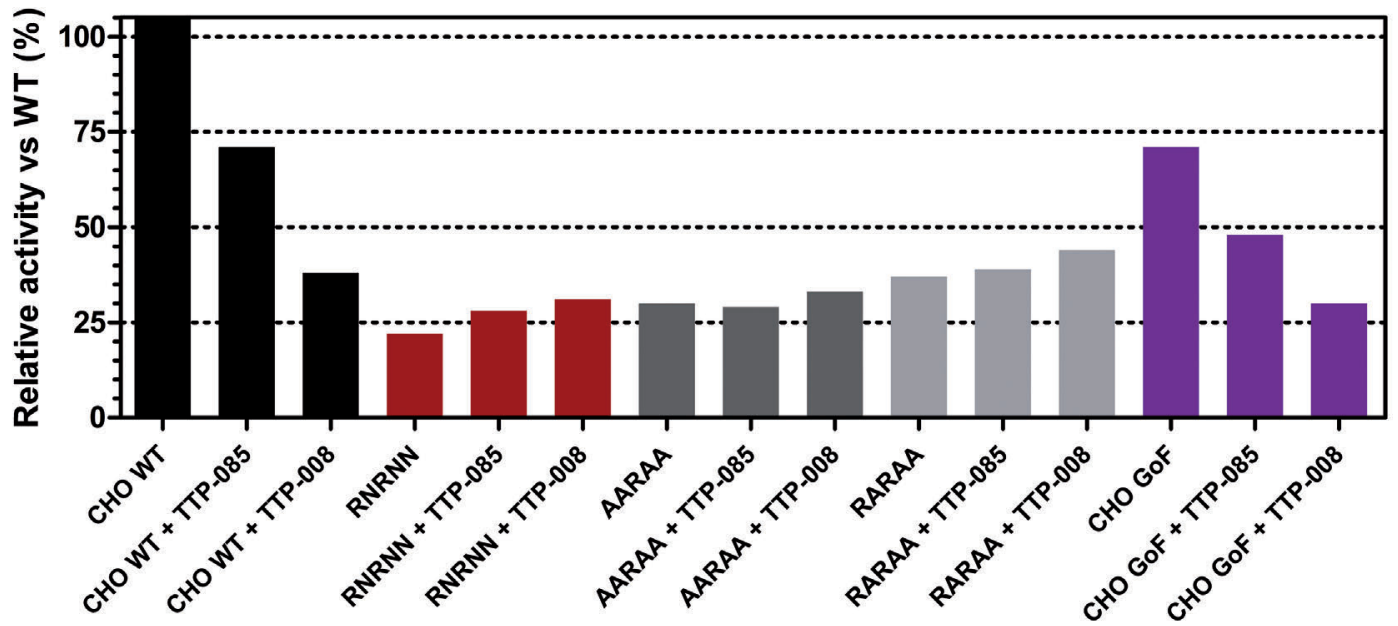

Figure 5. Patients' samples inhibit wild-type and gain-of-function (KYKFF) ADAMTS13 variants but not non-conservative or alanine ADAMTS13 variants. The activities of selected ADAMTS13 variants were tested in the presence of pathogenic patients' autoantibodies in FRETS-VWF73 assays. Three patients' samples, representative of the patients' autoantibody repertoire, were incubated directly with ADAMTS13 variants (each variant at $0.2 \mu \mathrm{g} / \mathrm{mL}$ ). (A). samples from TTP-007 and TTP008 (only anti-spacer autoantibodies) were diluted 40x. (B) Samples from TTP-008 (anti-spacer autoantibodies) and TTP-085 (anti-spacer and anti-C-terminal autoantibodies) were diluted 20x. As expected, both wild-type and gain-of-function variants were inhibited. Variants RNNN, AARAA and RARAA displayed full-resistance and retained their baseline proteolytic activity levels. WT: wild-type; CHO: Chinese hamster ovary; GoF: gain-of-function. 
latively mutated to either an asparagine or alanine. These mutations likely disrupt the hydrophobic cluster centered in F592 and formed together with Y661, Y665 and other neighboring residues. ${ }^{33}$ The greatest impact was achieved with cumulative mutations of all three aromatic residues, alone or together with mutations of the arginines. The highest resistance to autoantibody binding was observed for the full-length $5 \mathrm{x}$ ala mutant ( $\underline{\mathrm{AAAAA}})$, followed by the triple asparagine RNRNN mutant (Figure 2).

Protein surfaces enriched in tyrosine and arginine are usually involved in protein-protein interactions. Arginine salt-bridges are common players in these interactions. ${ }^{34}$ Tyrosine itself is a highly promiscuous residue participating in many types of interactions. ${ }^{35}$ This epitope contains two arginine residues, R568 and R660, which are likely involved in arginine-salt bridges for antibody binding (Figure 2), and also in intramolecular hydrogen bonds and cation- $\pi$ interactions with Y661 and Y665 (Online Supplementary Figure S5). Paratopes of the antibodies are considered to have a core enriched in aromatic residues, particularly tyrosine. ${ }^{36,37}$ The primary sequence of a large collection of anti-ADAMTS13 autoantibodies revealed that most autoantibodies targeting ADAMTS13 - encoded by heavy chain variable genes $V_{H} 1-69$ and $V_{H} 1-3^{11-13}-$ have a complementary determining region 3 (CDR3) consisting of multiple residues with either aromatic or negatively charged side chains. ${ }^{11,12}$ Exchanging a tyrosine for a phenylalanine (and vice versa) within exosite-3 conserves
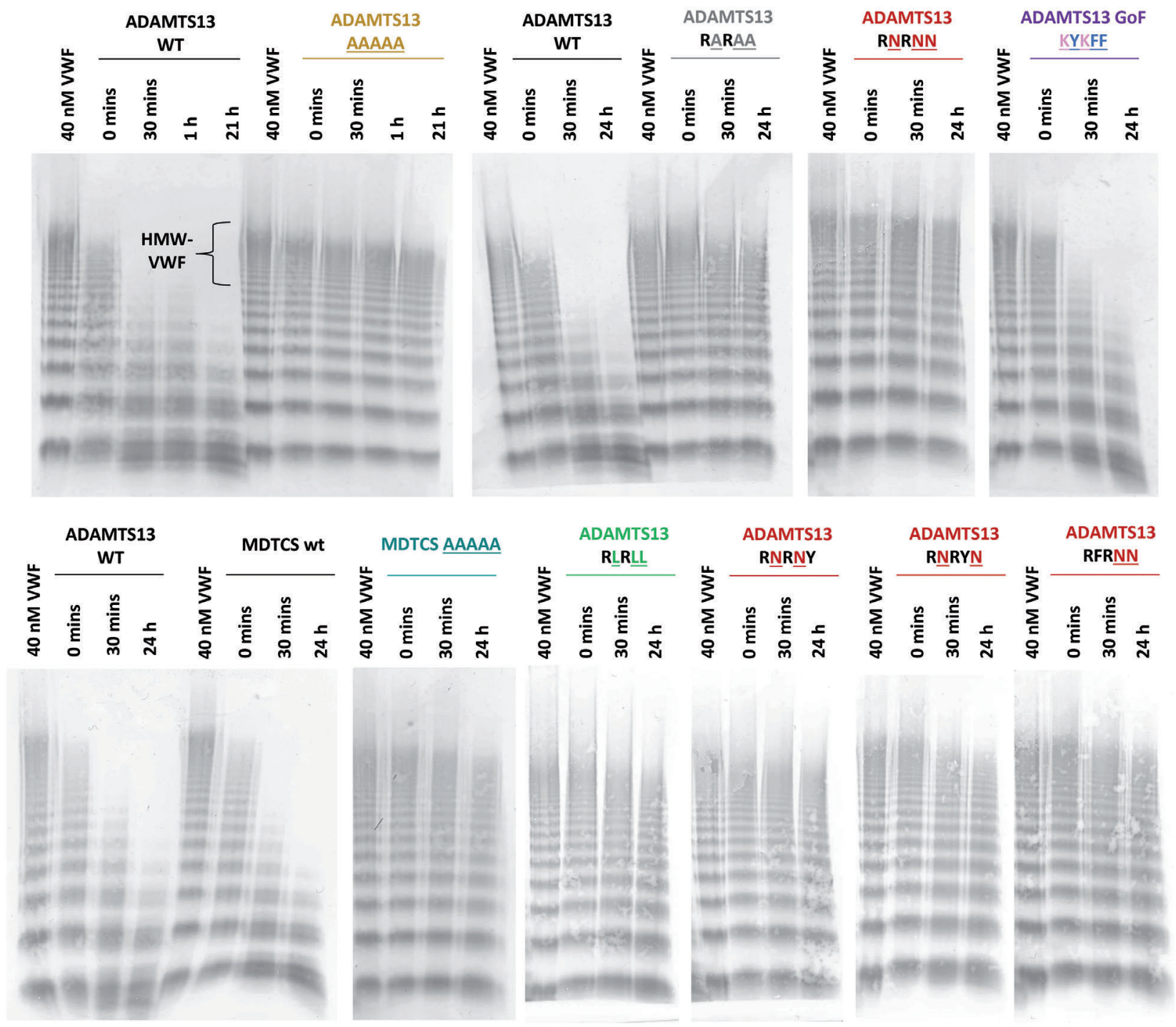

Figure 6. Autoantibody-resistant ADAMTS13 variants are capable of cleaving high-molecular weight multimers of von Willebrand factor. Selected variants with different levels of autoantibody reactivity/FRETS-VWF73 activity were tested for their proteolytic activity against recombinant multimeric von Willebrand factor (VWF) (1.9 nM ADAMTS13 for $40 \mathrm{nM} \mathrm{VWF)} \mathrm{in} \mathrm{a} \mathrm{static} \mathrm{assay} \mathrm{in} \mathrm{which} \mathrm{urea} \mathrm{was} \mathrm{used} \mathrm{to} \mathrm{denature} \mathrm{VWF} \mathrm{multimers.} \mathrm{The} \mathrm{full-length} \mathrm{wild-type} \mathrm{ADAMTS13} \mathrm{variant} \mathrm{was} \mathrm{used} \mathrm{as}$ a control for each individual reaction, with similar outcomes for all reactions. An example is shown in each panel for comparison. High-molecular weight VWF multimers are cleaved in a time-dependent manner, with accumulation of satellite bands in lower molecular weight regions. A lane containing 40 nM multimeric VWF without ADAMTS13 was included in the gels for comparison. All full-length variants displayed a reduction in proteolytic activity compared to the wild-type molecule. However, residual proteolytic activity was retained by several of them (including one of the most autoantibody-resistant variants, RARAA). The wild-type MDTCS and MDTCS-AAAAA were also tested, and while the wild-type MDTCS retained similar activity levels compared to the wild-type molecule, MDTCS-AAAAA displayed a reduction to the same level as its full-length counterpart, variant AAAAA. 
A

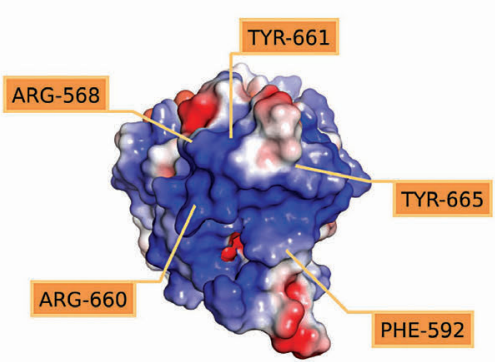

D

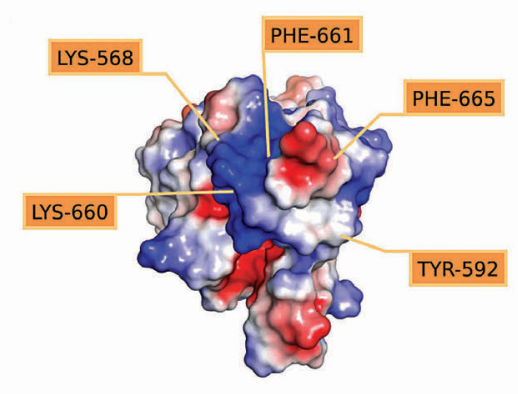

B

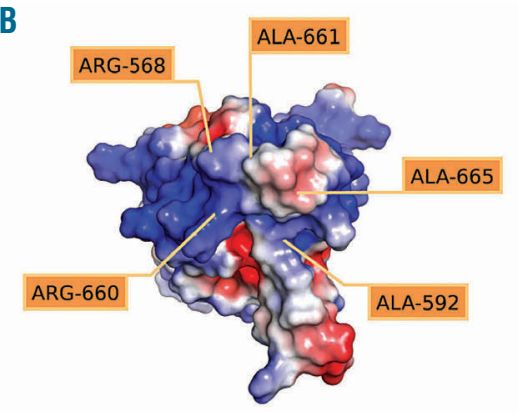

E

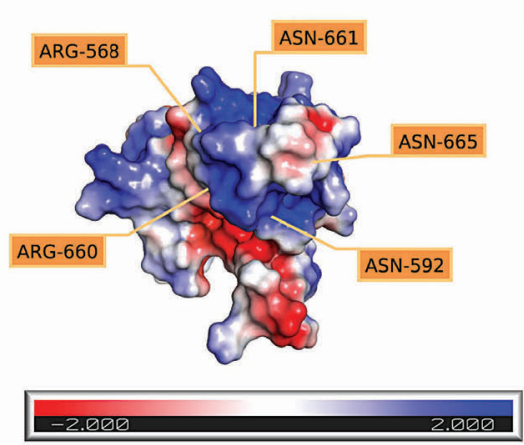

C

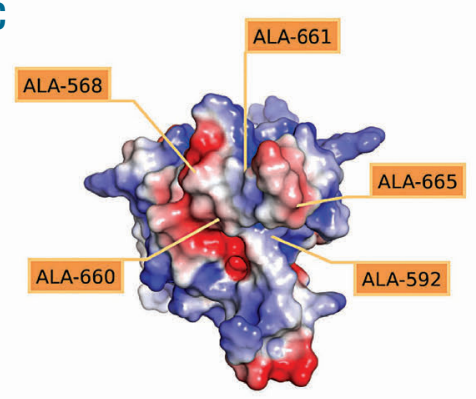

$\mathbf{F}$

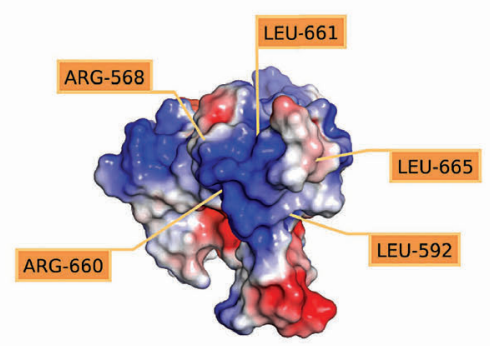

Figure 7. Models of three-dimensional structure and surface charge of ADAMTS13 spacer domain variants included in this study. (A-F) Three-dimensional structure model of the spacer domain of: (A) wild-type ADAMTS13 (RFRYY); (B) triple-alanine mutant variant (RARAA); (C). 5x alanine mutant variant (AAAAA); (D) gain-of-function variant (KYKFF); (E) triple-asparagine non-conservative mutant variant (RNRNN); and (F) semi-conservative variant (RLRLL). Negative charges are represented in red, neutral charges in white and positive charges in blue.

the aromatic ring and the capacity for establishing these intra- and other inter-molecular interactions. Replacement of arginines by lysines conserves the cationic charge in these positions. Our data clearly show that non-conservative arginine and alanine mutations in the ADAMTS13 spacer RFRYY epitope provide superior autoantibody resistance, likely because these mutations promote disruption of these intramolecular interactions and can potentially change the position of the loops of the epitope. We constructed three-dimensional models of selected epitope variants and these revealed reduction of total surface charge (Figure 7) and/or epitope surface size (Online Supplementary Figure S6).

The concept of ADAMTS13 variants that resist autoantibodies and remain active has been explored previously. However, the majority of previous studies designed with this intention were conducted with truncated ADAMTS13 variants, ${ }^{23,38}$ with the exception of the $\mathrm{GoF}^{26}$ and a few alanine variants. ${ }^{22}$ We present here for the first time a comprehensive study in which, in the context of the full length ADAMTS13 molecule, the spacer domain was modified with different types of mutations. We also demonstrated quantitatively that several full-length ADAMTS13 mutants escape the majority of autoantibodies from most patients. Therefore, we sought to assess whether these variants conserved proteolytic activity. The activities of ADAMTS13 variants have been measured in several forms of in vitro and in vivo assays. Evidence strongly suggests that the C-terminal TSP2-8/CUB1-2 domains of ADAMTS13 are important for full in vivo proteolytic activity ${ }^{39,40}$ because these domains allow ADAMTS13 to anchor itself onto the D4-CK domains of full-length VWF under shear flow conditions. ${ }^{41,42}$ The static FRETS-VWF73 assay is commonly used to measure the activity of ADAMTS13 for the diagnosis of
TTP. ${ }^{31}$ In minimal peptide assays, however, it appears that the C-terminal domains of ADAMTS13 and the spacer domain itself are less relevant for maintaining activity. ${ }^{43}$ In FRETS-VWF73 the activity of the MDTCS fragment is higher than that of full-length ADAMTS13 ${ }^{32,44}$ due to the lack of the terminal TSP2-8 and CUB1-2 domains, eliminating their auto-inhibitory properties. ${ }^{44}$ Our data are in agreement with these earlier findings: both the wild-type MDTCS and the MDTCS-AAAAA variant had higher activity in the FRETS-VWF73 assay compared to their fulllength counterparts. In agreement with previous studies most alanine changes induced loss of activity. ${ }^{22,23,25}$ Our data suggest that both aromatic residues and arginine residues have similar importance for activity. We observed a clear inverse correlation between resistance to autoantibodies and proteolytic activity of the variants (Figure 4). Selected mutants tested in our VWF multimer assay also had lower activity than the wild-type molecule, with the exception of wild-type MDTCS. This highlights the functional importance of the spacer exosite-3 in our VWF multimer assay. Among the selected full-length autoantibody-resistant mutants, the RARAA mutant demonstrated residual activity in conditions of sub-physiological ADAMTS13 concentrations (Figure 6). This variant was tested in FRETSVWF73 together with patients' autoantibodies (Figure 5) exhibiting full resistance to the inhibitory action of polyclonal autoantibodies from patients.

Surprisingly, the GoF variant described previously showed less activity than that of wild-type ADAMTS13. The GoF variant was originally characterized in a COS-7 cell background ${ }^{26}$ and in all subsequent in vitro ${ }^{32,44,45}$ and in vivo studies $^{46,47}$ it was reported to be more active than wild-type ADAMTS13. We are the first group observing a loss of function of this molecule. The reasons for this are not clear. 
It is possible that the differences observed may be due to post-translational modifications, such as different glycosylation patterns that may result from the CHO-based transient expression system that we used in our study. ${ }^{48,49}$ To address this we also expressed the GoF variant in HEK293 cells, and under these conditions the GoF variant displayed increased activity, compared to the respective HEK293 wild-type ADAMTS13, in both FRETS-VWF73 and in the VWF multimer assay (Online Supplementary Figure S7). In the context of our ELISA, the GoF variant expressed in both $\mathrm{CHO}$ and HEK293 cells behaved in a similar way against the patients' samples (Online Supplementary Figure S7), and importantly our CHO-produced GoF variant was also inhibited by patients' autoantibodies (Figure 5), as anticipated by our ELISA results (Figure 2).

In conclusion, we have shown that less conservative mutations make it possible to obtain ADAMTS13 variants that resist the majority of patients' autoantibodies and retain residual levels of activity in vitro. Our data suggest a trade-off between the resistance towards patients' autoantibody binding and the loss of proteolytic activity in spacer exosite-3 ADAMTS13 variants. The study also reveals the importance of the side-chains of the RFRYY epitope in the spacer domain for autoantibody binding and ADAMTS13 activity, and it provides a basis for the development of novel therapeutic interventions that prevent the binding of pathogenic autoantibodies to the spacer domain of ADAMTS13.

\section{Acknowledgments}

This project received funding from the European Union's Horizon 2020 research and innovation program under the Marie Skłodowska-Curie grant agreement number 675746 (PROFILE). The authors would like to thank Tiiu Männik, Kristiina Karro, Urve Toots (Icosagen AS), Elien Roose (KU Leuven) and Ellie Karampini (Sanquin) for their technical advice, and to all the patients from CNR-MAT who consented to the use of their materials.

\section{References}

1. Kremer Hovinga JA, Coppo P, Lämmle B, Moake JL, Miyata T, Vanhoorelbeke K. Thrombotic thrombocytopenic purpura. Nat Rev Dis Prim. 2017;3:17020.

2. Zheng $X$, Chung D, Takayama TK, Majerus EM, Sadler JE, Fujikawa K. Structure of von Willebrand factor-cleaving protease (ADAMTS13), a metalloprotease involved in thrombotic thrombocytopenic purpura. J Biol Chem. 2001:276(44):41059-41063

3. Hrdinová J, D’Angelo S, Graça NAG, et al. Dissecting the pathophysiology of immune thrombotic thrombocytopenic purpura: interplay between genes and environmental triggers. Haematologica. 2018;103(7):10991109

4. Booth KK, Terrell DR, Vesely SK, George JN. Systemic infections mimicking thrombotic thrombocytopenic purpura. Am J Hematol. 2011;86(9):743-751

5. Koh YR, Hwang SH, Chang CL, Lee EY, Son $\mathrm{HC}$, Kim HH. Thrombotic thrombocytopenic purpura triggered by influenza A virus subtype H1N1 infection. Transfus Apher Sci. 2012;46(1):25-28.

6. Medina PJ, Sipols JM, George JN. Drug-associated thrombotic thrombocytopenic purpura-hemolytic uremic syndrome. Curr Opin Hematol. 2001;8(5):286-293.

7. Ashley JR, Otero H, Aboulafia DM. Bee envenomation: a rare cause of thrombotic thrombocytopenic purpura. South Med J. 2003;96(6):588-591

8. Valavi E, Ahmadzadeh A, Amoori P, Daneshgar A. High frequency of acquired ADAMTS13 deficiency after hemolysis in Hemiscorpius lepturus (scorpion) stung children. Indian J Pediatr. 2014;81(7):665-669.

9. Pos W, Luken BM, Sorvillo N, Hovinga JAK, Voorberg J. Humoral immune response to ADAMTS13 in acquired thrombotic thrombocytopenic purpura. J Thromb Haemost. 2011:9(7):1285-1291.

10. Ostertag EM, Kacir S, Thiboutot M, et al. ADAMTS13 autoantibodies cloned from patients with acquired thrombotic thrombocytopenic purpura: 1 . Structural and functional characterization in vitro. Transfusion. 2016;56(7):1763-1774.
11. Pos W, Luken BM, Kremer Hovinga JA, et al. VH1-69 germline encoded antibodies directed towards ADAMTS13 in patients with acquired thrombotic thrombocytopenic purpura. J Thromb Haemost. 2009; 7(3):421-428.

12. Schaller M, Vogel M, Kentouche K, Ammle B, Kremer Hovinga JA. The splenic autoimmune response to ADAMTS13 in thrombotic thrombocytopenic purpura contains recurrent antigen-binding CDR3 motifs. Blood. 2014;124(23):3469-3479.

13. Luken BM, Kaijen PHP, Turenhout EAM, et al. Multiple B-cell clones producing antibodies directed to the spacer and disintegrin/thrombospondin type-1 repeat 1 (TSP1) of ADAMTS13 in a patient with acquired thrombotic thrombocytopenic purpura. J Thromb Haemost. 2006;4(11):23552364

14. Luken BM, Turenhout EAM, Hulstein JJJ, Van Mourik JA, Fijnheer R, Voorberg J. The spacer domain of ADAMTS13 contains a major binding site for antibodies in patients with thrombotic thrombocytopenic purpura. Thromb Haemost. 2005;93(2):267-274.

15. Klaus C, Plaimauer B, Studt JD, et al. Epitope mapping of ADAMTS13 autoantibodies in acquired thrombotic thrombocytopenic purpura. Blood. 2004;103(12):4514-4519.

16. Long Zheng X, Wu HM, Shang D, et al Multiple domains of ADAMTS13 are targeted by autoantibodies against ADAMTS13 in patients with acquired idiopathic thrombotic thrombocytopenic purpura Haematologica. 2010;95(9):1555-1562.

17. Thomas MR, de Groot R, Scully MA, Crawley JTB. Pathogenicity of antiADAMTS13 autoantibodies in acquired thrombotic thrombocytopenic purpura. EBioMedicine. 2015;2(8):942-952

18. Casina VC, Hu W, Mao J-H, Lu R-N, Hanby HA, Pickens B, et al. High-resolution epitope mapping by HX MS reveals the pathogenic mechanism and a possible therapy for autoimmune TTP syndrome. Proc Natl Acad Sci U S A. 2015;112(31):9620-9625.

19. Scheiflinger F, Knöbl P, Trattner B, et al. Nonneutralizing $\operatorname{IgM}$ and $\operatorname{Ig} G$ antibodies to von Willebrand factor-cleaving protease (ADAMTS-13) in a patient with thrombotic thrombocytopenic purpura. Blood. 2003;102 (9):3241-3243.
20. Shelat SG, Smith P, Ai J, Zheng XI. Inhibitory autoantibodies against ADAMTS-13 in patients with thrombotic thrombocytopenic purpura bind ADAMTS13 protease and may accelerate its clearance in vivo. J Thromb Haemost. 2006;4(8):17071717.

21. Yamaguchi Y, Moriki T, Igari A, et al Epitope analysis of autoantibodies to ADAMTS13 in patients with acquired thrombotic thrombocytopenic purpura. Thromb Res. 2011;128(2):169-173.

22. Pos W, Crawley ITB, Fijnheer R, Voorberg J, Lane DA, Luken BM. An autoantibody epitope comprising residues R660, Y661, and Y665 in the ADAMTS13 spacer domain identifies a binding site for the $\mathrm{A} 2$ domain of VWF. Blood. 2010;115(8):1640-1649.

23. Pos W, Sorvillo N, Fiinheer R, et al. Residues arg568 and phe592 contribute to an antigenic surface for anti-ADAMTS13 antibodies in the spacer domain. Haematologica. 2011;96(11):1670-1677

24. Luken BM, Turenhout EAM, Kaijen PHP, et al. Amino acid regions 572-579 and 657-666 of the spacer domain of ADAMTS13 provide a common antigenic core required for binding of antibodies in patients with acquired TTP. Thromb Haemost. 2006;96 (3):295-301

25. Jin S-Y, Skipwith CG, Zheng XL. Amino acid residues Arg659, Arg660, and Tyr661 in the spacer domain of ADAMTS13 are critical for cleavage of von Willebrand factor. Blood. 2010;115(11):2300-2310

26. Jian C, Xiao J, Gong L, et al. Gain-of-function ADAMTS13 variants that are resistant to autoantibodies against ADAMTS13 in patients with acquired thrombotic thrombocytopenic purpura. Blood. 2012;119(16) 3836-3843

27. Roose E, Schelpe A-S, Joly BS, et al. An open conformation of ADAMTS-13 is a hallmark of acute acquired thrombotic thrombocytopenic purpura. J Thromb Haemost. 2018;16(2):378-388

28. Schelpe A-S, Roose E, Joly BS, et al Generation of anti-idiotypic antibodies to detect anti-spacer antibody idiotopes in acute thrombotic thrombocytopenic purpura patients. Haematologica. 2018;104(6) 1268-1276. 
29. Feys HB, Roodt J, Vandeputte N, et al. Thrombotic thrombocytopenic purpura directly linked with ADAMTS13 inhibition in the baboon (Papio ursinus). Blood. 2010;116(12):2005-2010.

30. Alwan F, Vendramin C, Vanhoorelbeke K, et al. Presenting ADAMTS13 antibody and antigen levels predict prognosis in immunemediated thrombotic thrombocytopenic purpura. Blood. 2017;130(4):466-471.

31. Kokame K, Nobe Y, Kokubo Y, Okayama A, Miyata T. FRETS-VWF73, a first fluorogenic substrate for ADAMTS13 assay. Br J Haematol. 2005;129(1):93-100.

32. Muia J, Zhu J, Gupta G, et al. Allosteric activation of ADAMTS13 by von Willebrand factor. Proc Natl Acad Sci U S A. 2014;111 (52):18584-18589.

33. Akiyama M, Takeda S, Kokame K, Takagi J, Miyata T, Majerus PW. Crystal structures of the noncatalytic domains of ADAMTS13 reveal multiple discontinuous exosites for von Willebrand factor. Proc Natl Acad Sci U S A. 2009;106(46):19274-19279.

34. Bogan AA, Thorn KS. Anatomy of hot spots in protein interfaces. J Mol Biol. 1998;280 (1):1-9.

35. Ferreira De Freitas R, Schapira M. A systematic analysis of atomic protein-ligand interactions in the PDB. Medchemcomm. 2017:8(10):1970-1981.

36. Robin G, Sato Y, Desplancq D, Rochel N, Weiss E, Martineau P. Restricted diversity of antigen binding residues of antibodies revealed by computational alanine scanning of 227 antibody-antigen complexes. J Mol Biol. 2014;426(22):3729-3743

37. Peng H-P, Lee KH, Jian J-W, Yang A-S. Origins of specificity and affinity in antibody-protein interactions. Proc Natl Acad Sci U S A. 2014;111(26):E2656-E2665

38. Zhou W, Dong L, Ginsburg D, Bouhdssira EE, Tsai HM. Enzymatically active ADAMTS13 variants are not inhibited by anti-ADAMTS13 autoantibodies: a novel therapeutic strategy? J Biol Chem. 2005;280 (48):39934-39941

39. Banno F, Chauhan AK, Kokame K, et al. The distal carboxyl-terminal domains of ADAMTS13 are required for regulation of in vivo thrombus formation. Blood. 2009;113 (21):5323-5329.

40. Zhu J, Muia J, Gupta G, et al. Exploring the "minimal" structure of a functional ADAMTS13 by mutagenesis and smallangle X-ray scattering. Blood. 2019;133(17): 1909-1918.

41. Zhang P, Pan W, Rux AH, Sachais BS, Zheng $\mathrm{XL}$. The cooperative activity between the carboxyl-terminal TSP1 repeats and the CUB domains of ADAMTS13 is crucial for recognition of von Willebrand factor under flow. Blood. 2007;110(6):1887-1894.

42. Crawley ITB, De Groot R, Xiang Y, Luken BM, Lane DA. Unraveling the scissile bond: how ADAMTS13 recognizes and cleaves von Willebrand factor. Blood. 2011:118(12): 3212-3221

43. Petri A, Kim HJ, Xu Y, et al. Crystal structure and substrate-induced activation of ADAMTS13. Nat Commun. 2019;10(1):116.

44. South K, Luken BM, Crawley ITB, et al. Conformational activation of ADAMTS13. Proc Natl Acad Sci U S A. 2014;111(52): 18578-18583.

45. South K, Freitas MO, Lane DA Conformational quiescence of ADAMTS-13 prevents proteolytic promiscuity. J Thromb Haemost. 2016;14(10):2011-2022.

46. South K, Denorme F, Salles-Crawley II, De Meyer SF Lane DA. Enhanced activity of an ADAMTS-13 variant (R568K/F592Y/ R660K/Y661F/Y665F) against platelet agglutination in vitro and in a murine model of acute ischemic stroke. J Thromb Haemost. 2018;16(11):2289-2299.

47. Liu-Chen S, Connolly B, Cheng L, Subramanian RR, Han Z. MRNA treatment produces sustained expression of enzymatically active human ADAMTS13 in mice. Sci Rep. 2018;8(1):7859.

48. Goh JB, Ng SK. Impact of host cell line choice on glycan profile. Crit Rev Biotechnol. 2018;38(6):851-867

49. Werner RG, Kopp K, Schlueter M Glycosylation of therapeutic proteins in different production systems. Acta Paediatr. 2007;96(455):17-22. 